\title{
DESIGNING A TEMPERATURE MNITORING AND CONTROL SYSTEM IN AN ECLOSURE USING PELTIER ELEMENTS
}

\author{
Monica-Anca Chita \\ University of Pitesti, Pitesti, Street Targu din Vale, No. 1, 110040, Romania. \\ *monica.chita@upit.ro and chita_monica@yahoo.com
}

\begin{abstract}
The paper presents a series of considerations regarding the designing a temperature monitoring and control system in an enclosure using Peltier elements. A $36 \mathrm{~W}$ Peltier element, which can reach a temperature difference of $68{ }^{\circ} \mathrm{C}$, having a maximum working temperature of $138{ }^{\circ} \mathrm{C}$, was used. The power supply of this element will be directly from a $12 \mathrm{~V}$ voltage source with a maximum working voltage of $14.6 \mathrm{~V}$ and a maximum working current of 4.3 A. For the best effect of Peltier's element cooling effect on the enclosure, it is necessary that the lower part of the element, where the radiator and cooler are located to prevent the Peltier element from overheating and get burnt, another method of cooling (with liquid) must be used. There is a difference in temperature between the two sides and the performances of the Peltier element. For a more accurate reading of the temperature from the enclosure, a temperature transducer can be used, that is at least two sensors to read the temperature from several different parts of the enclosure it is possible to mediate the temperature readings and, in this way, the displayed temperature will be closer to the temperature existent in the enclosure.
\end{abstract}

Keywords: Engineering, Temperature monitoring, Control system, Enclosure, Peltier elements.

\section{Introduction}

Thermoelectric effects typically occur at the contact between semiconductor materials of different nature, and consist of the appearance of a thermo- electromotive tension / voltage (the Seebeck effect), when a temperature gradient is established between the materials in contact, or the occurrence of a temperature difference (the Peltier effect), when an electric current circulates through these junctions.

Semiconductor materials such as $\mathrm{Si}$ and Ge have two types of electric charge carriers, electrons and voids. The doping densities of the semiconductor elements vary from a maximum value in the area of the hot sides, as it can be seen in Figure 1, to a lower density in the area of the cold sides. An electrical current is applied by a series of semiconductor elements of type $n$ and $p$ so that the electric charge carriers will move in the same direction, as it can be seen in Fig. 1, from high density areas, to areas with low doping rates.

This causes the electron gas, reaching the weak doping area, to suffer an adiabatic expansion process, similar to that of an ordinary gas, causing its temperature to drop. The same process will also characterize the gas formed by the voids. In order to increase the efficiency of such a process, several elements are connected in series, such as those shown in FIG. 1 so that the hot and cold sides are in thermal contact, as it is shown in fig. 2, where a Peltier element battery is illustrated.

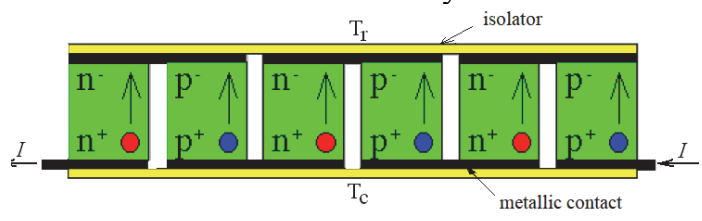

Figure 1. Peltier element battery.

Therefore, such a device can be regarded as a plate of a $d$ value thickness, which carries the heat from the cold side, of a $\mathrm{Tr}$ temperature, to the hot side, of a $T c$ temperature. The balance of powers in such a structure is shown in fig. 2 . 


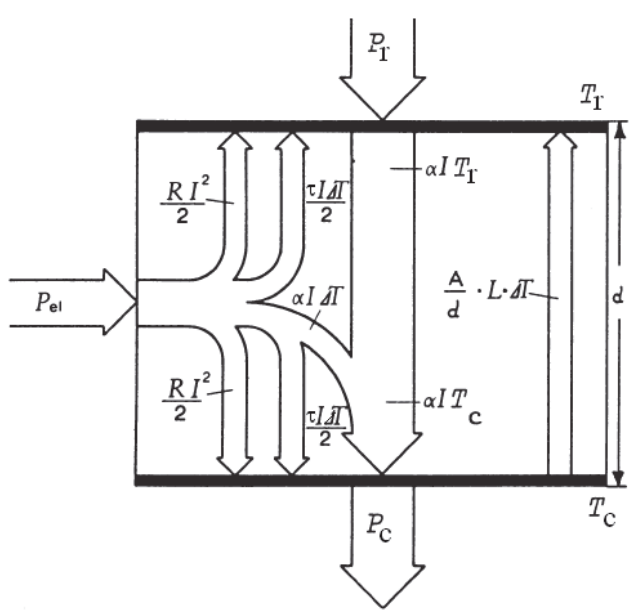

Figure 2. Balance of powers in a Peltier element battery.

In the paper, a $36 \mathrm{~W}$ Peltier element was used, which can reach a temperature difference of $68{ }^{\circ} \mathrm{C}$, having a maximum working temperature of $138{ }^{\circ} \mathrm{C}$. The power supply of this element will be directly from a $12 \mathrm{~V}$ voltage source, with a maximum working voltage of $14.6 \mathrm{~V}$ and a maximum working current of $4.3 \mathrm{~A}$.

\section{Designing the Block Diagram}

The block diagram of the projected system is shown in fig. 3 .

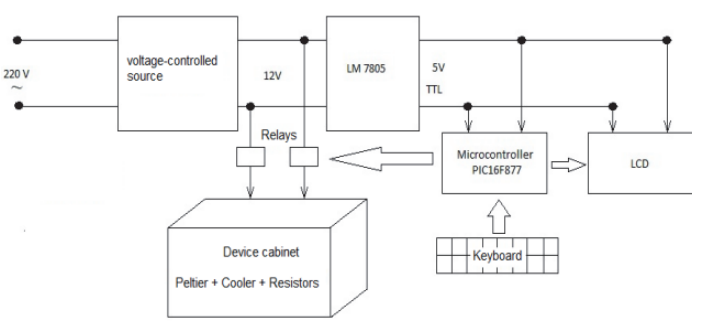

Figure 3. Block diagram.

Starting from left to right it will be noticed that on the left side we have $220 \mathrm{~V}$, alternative voltage resulted from the network. This voltage will power up the controlled switching power source, which will output a voltage equal to $12 \mathrm{~V}$ (continuous voltage), with which we will then power up the elements in the enclosure, more precisely, we will power up our Peltier element, the 4 resistors connected in series, with a value of $10 \mathrm{~W}$ each (used to heat the enclosure), we will also power up a $12 \mathrm{~V}$ cooler (which is designed to equalize the temperature inside the enclosure / device cabinet).

Also, at the $12 \mathrm{~V}$ output (continuous voltage), an integrated LM7805 circuit will be connected, which will convert the $12 \mathrm{~V}$ into $5 \mathrm{~V}$, used to power up the PIC16F877 microcontroller and also to power up an LCD $2 \mathrm{X} 16$.

This microcontroller was chosen because it required a large number of pins (in/out), this having 40 pins, out of which 33 were used. The electrical pattern resulting from the design is shown in fig. 4 . The solution would be to have a software to track both the pressing of a key as well as the decoupling of that key, as it can be seen in fig. 5 .

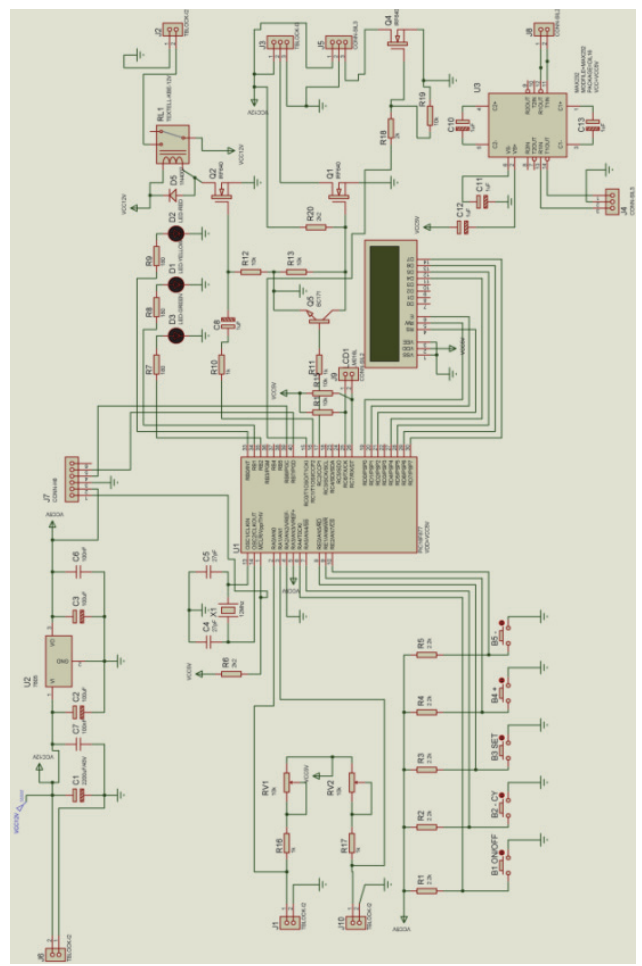

Figure 4. Electrical scheme.

\section{System Softwae Designing}

Introduction to $\mathrm{C}^{++}$Programing Software

Microprocessor programming was done in $\mathrm{C}^{++}$. $\mathrm{A} \mathrm{C}^{++}$written program consists of one or more source files. A source file is a text file containing the source code (in the C language) of a program. Each source file contains one or more functions, and possibly references to one or more header files (fig. 6). The main function of a program is called main. 


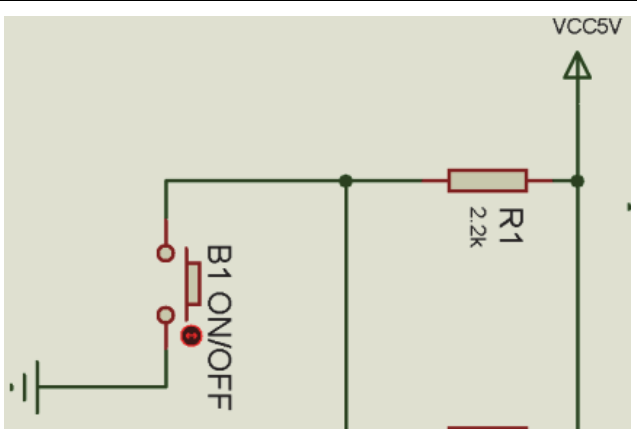

Figure 5. Connecting the dip key button to PIC16F877.

The execution of the program starts with the execution of this function, which can also call, in its turn, for other functions. The actions in each function are encoded by instructions (Figure 6.1.a.). An instruction is any valid expression (usually an assignment or a function call) followed by the ";" symbol. An instruction is any valid expression (usually an assignment or a function call) followed by the ";" symbol. In fig. 6.1.b. an example of a simple instruction is given.

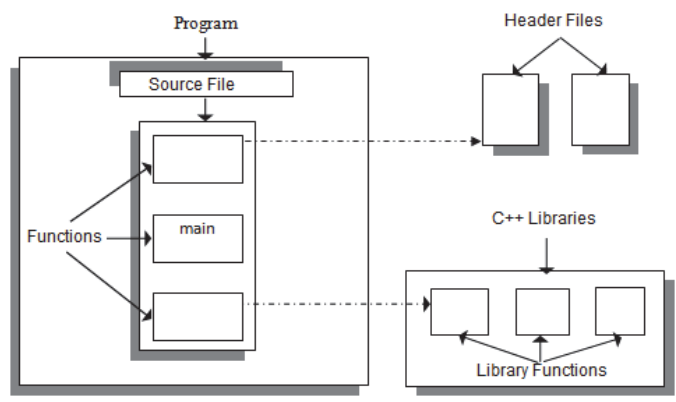

Figure 6. Structure of a program in the $\mathrm{C}$ language.

\section{The Diagram Designing}

The diagrams used for the system software design are presented below. Thus, in fig. 7 there is a diagram corresponding to the cooling function:

Fig. 8 shows the diagram corresponding to the reading function of the int1 sensor: In fig. 9 there is a diagram corresponding to the keypad reading function (a key).

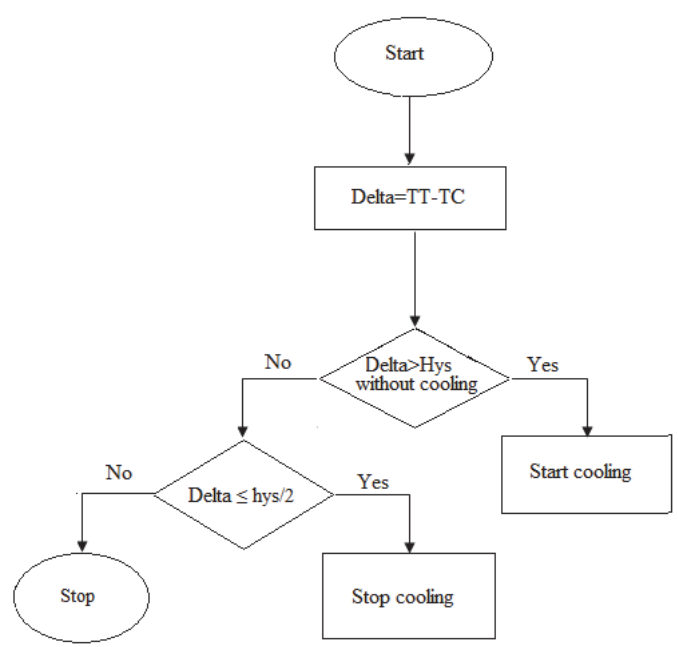

Figure 7. Cooling function diagram.

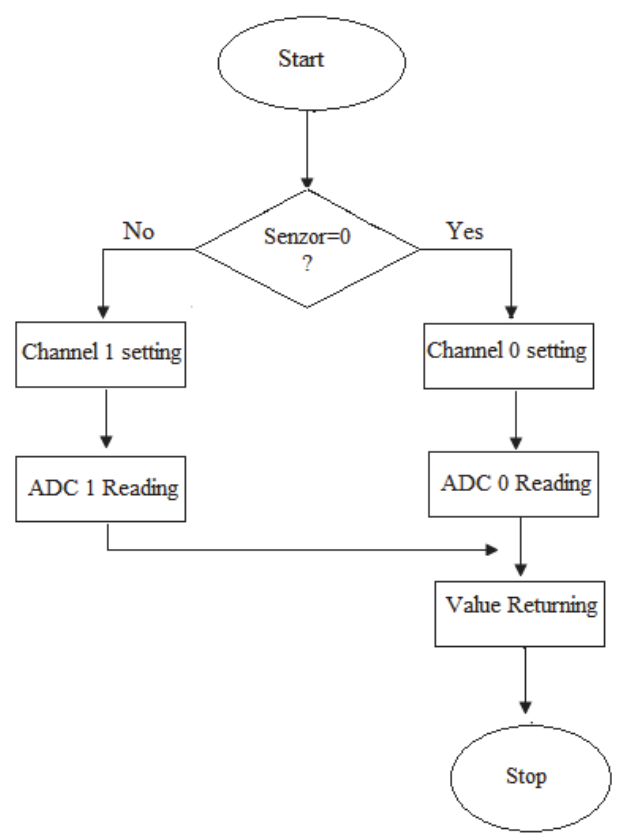

Figure 8. Diagram corresponding to the int 1 sensor reading function.

Observtions and Interpretations on Enclosure Temperature Monitoring

Use the International System of Units (SI) only. Never combine SI units and CGS or other units. If other units are unavoidable, make sure it can't be misinterpreted. 


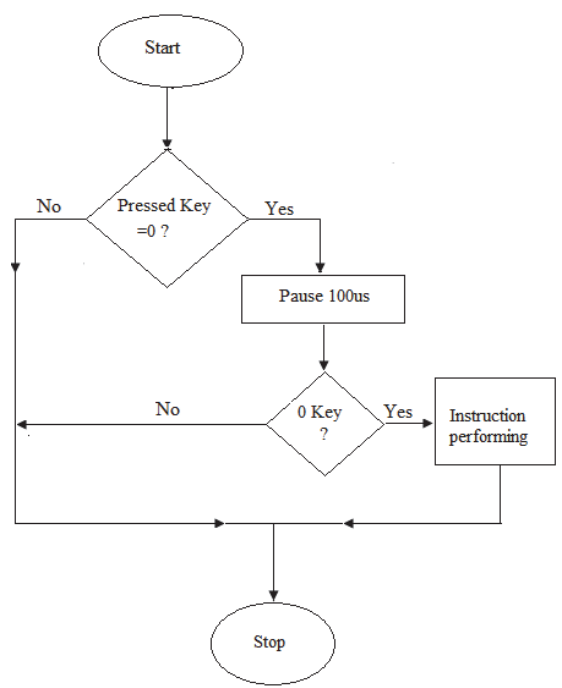

Figure 9. Diagram corresponding to the keyboard reading function.

\section{How to Create the Layout}

After the electrical scheme is done with the help of the Proteus ISIS program, and subsequently the PCB with the Proteus ARES program, a laser printer is used to print the wiring. Theoretically, the wiring can be printed on any type of sheet, but in practice it requires a special sheet used in electronics with a glossy side (as seen in the photo); the scheme printed on this special sheet is shown in fig. 10. After the scheme was printed on this paper sheet, it is possible to go to the next step, namely to print the scheme on the wiring harness. After printing the scheme on this paper sheet, move on to the next step, i.e. to print the scheme on the wiring harness. After printing the wires on the wiring harness, remove the ink from the wiring, which is to be done carefully in order to avoid interrupting any path. The next step will be to start the drilling of the plywood in the signed locations, and then move to the placement and applying of the pieces on the plate, as it can be seen in fig. 11 .

Making drawings on the upper (top) side of the wiring harness is the same as the one on the copper side only that, in order to fit it, it is necessary to apply the mirror command to the electronic tracks, as it can be seen in fig. 12 .

There is an enclosure made of glass, which is laid with the bottom up, and at the bottom side it is supported by a metal sheet. The sensor located in the enclosure transmits a temperature value to the microcontroller, which in turn displays it on an LCD, as it can be seen in fig. 13.

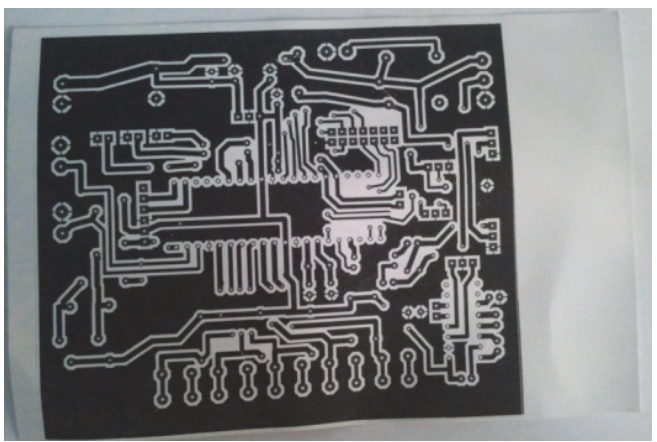

Figure 10. Wiring scheme.

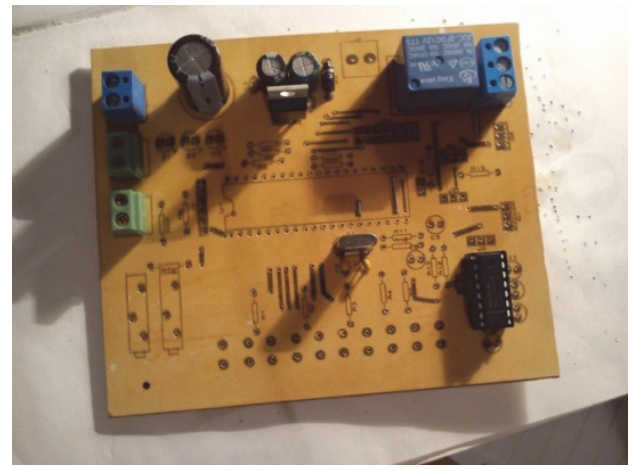

Figure 11. Electronic layout under construction.

In the figure above the device is in the off mode and the $\mathrm{Tc} 1$ sensor reads a temperature of 28.0 degrees Celsius, $\mathrm{P}=0$ is the power applied to the resistors when the enclosure is heated. The active heating mode is shown in Figure 13 since the target temperature is $\mathrm{TT}=$ $35.0^{\circ} \mathrm{C}$ and the current temperature is $\mathrm{Tc} 1=$ $32.2^{\circ} \mathrm{C}$. Selection of the sensor is done, as shown in fig. 14.

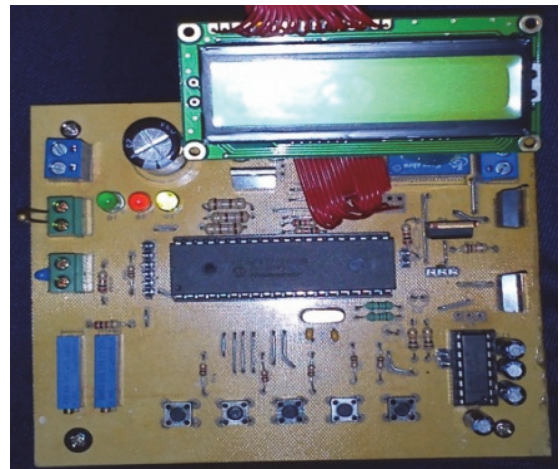

Figure 12. Electronic layout final result. 


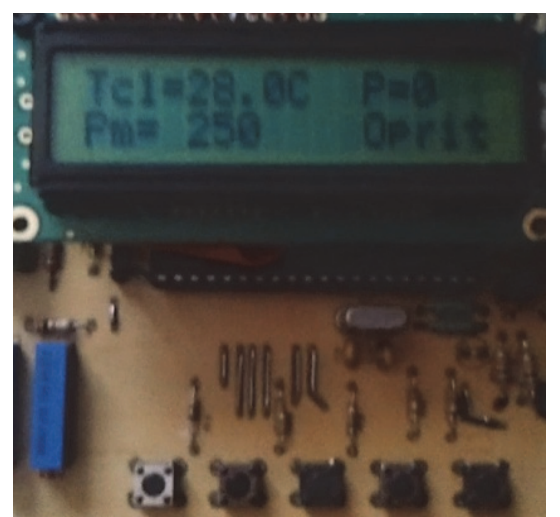

Figure 13. Off mode.

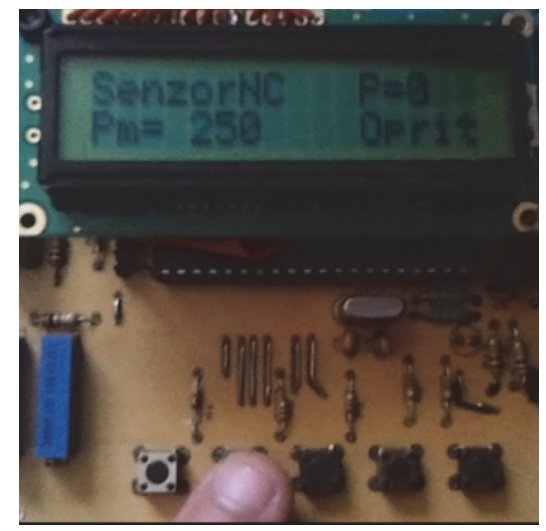

Figure 14. Sensor selecting.

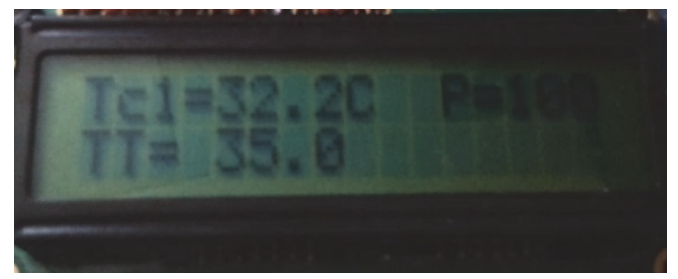

Figure 15. Active heating mode.

The cooler mode, shown in Figure 14, indicates the fact that the current temperature is $\mathrm{Tc} 1=31.2^{\circ} \mathrm{C}$; it is desired that the $\mathrm{TT}$ temperature is $\mathrm{TT}=20.0^{\circ} \mathrm{C}$

\section{Conclusions}

For the best effect of Peltier's cooling effect on the enclosure, it is necessary that the lower part of the peltier (the heater part) where the radiator and cooler are located, in order to prevent the Peltier element from overheating and burning, another coolant method (liquid based) should be used, in which case the temperature cannot be lowered under the ambient temperature. Between the two sides there is a temperature difference on which the performance of the Peltier element also depends.

Semiconductor materials such as $\mathrm{Si}$ and Ge have two types of charge carriers, electrons and voids. If it is desired that the enclosure should be perfectly thermally stable, it is necessary that the walls of the enclosure to be made of the best thermal insulation materials with a minimum heat dissipation coefficient, in addition, in this case the lower part of the enclosure is made of metal sheet which quickly dissipates the temperature. Initially, a larger volume enclosure was used that was not insulated with anything, then the enclosure was modified (hence it was reduced) and the thermal insulation with polyester was applied in an improvement attempt.

To find out exactly the temperature in the enclosure, you can use at least two sensors instead of a temperature sensor to read the temperature from several parts of the enclosure (if we have a sensor and that sensor reads a wrong temperature due to a current or for other reasons), we may also encounter apply an average value of the readings and this way the displayed temperature will be closer to the temperature inside the enclosure.

\section{References}

G. V. Cimpoca, Iulian Bancuta, "Physics and Technology of Thermoelectric Materials", Bibliotheca Edition, Targoviste, Romania, 2007.

C. Balan Radu, "Microcontrollers - Structure and Applications", Edition Todesco, ClujNapoca, Romania, 2002.

***Microsoft, Qbasic language help.

M. Bodea, "Integrated Linear Circuits, User Manual", Tehnica Edition, Bucharest, 1980.

***Online Course - PIC Microcontrolleres.

***Microchip PIC16F877, Datasheet, 2016.

D. Rotar, "Microprocessors" Course, Edition Alma Mater, Bacau, 2007.

***http: //en.innovati.com.tw/down/html/ LCD2x16A_En_v100.pdf

****http: $\quad-/ /$ www.drotar.lx.ro

"Microprocessor Course". 